\title{
Electromagnetic Wave Propagation in lonospheric Plasma
}

\author{
Ali Yeşil ${ }^{1}$ and İbrahim Ünal ${ }^{2}$ \\ ${ }^{1}$ Firat University, Elazı̆̆ \\ 2İnönü University, Malatya \\ Turkey
}

\section{Introduction}

Ionosphere physics is related to plasma physics because the ionosphere is, of course, a weak natural plasma: an electrically neutral assembly of ions and electrons [1]. The ionosphere plays a unique role in the Earth's environment because of strong coupling process to regions below and above [2]. The ionosphere is an example of naturally occurring plasma formed by solar photo-ionization and soft x-ray radiation. The most important feature of the ionosphere is to reflect the radio waves up to $30 \mathrm{MHz}$. Especially, the propagation of these radio waves on the HF band makes the necessary to know the features and the characteristics of the ionospheric plasma media. Because, when the radio waves reflect in this media, they are reflected and refracted depending on their frequency, the frequency of the electrons in the plasma and the refractive index of the media and thus, they are absorbed and reflected by the media.

The understanding of the existence of a conductive layer in the upper atmosphere has been emerged in a century ago. The idea of a conductive layer affected by the variations of the magnetic field in the atmosphere has been put forward by the Gauss in 1839 and Kelvin in 1860. Newfoundland radio signal from Cornwall to be issued by Marconi in 1901, at the first experimental evidence of the existence of ionospheric, respectively. In 1902, Kennely and Heaviside indicated that the waves are reflected from a conductive layer on the upper parts of the atmosphere. In 1902, Marconi stated that changing the conditions of night and day spread. In 1918, high-frequency band has been used by aircraft and ships. HF band in the 1920s has increased the importance of expansion. Then, put forward the theory of reflective conductive region, has been shown by experiments made by Appleton and Barnet. The data from the 1930s started to get clearer about the ionosphere and The Radio Research Station, Cavendish Laboratory, the National Braun of Standards, the various agencies such as the Carnegie Institution began to deal with the issue. In the second half of the 20th century, the work of the HF electromagnetic wave has been studied by divided into three as the fullwave theory, geometrical optics and conductivity. Despite initiation of widespread use of satellite-Earth communication systems, the use of HF radio spectrum for civilian and military purposes is increasing. Collapse of communication systems, especially in case of emergency situations, communication is vital in this band.

In the ionosphere, a balance between photo-ionization and various loss mechanisms gives rise to an equilibrium density of free electrons and ions with a horizontal stratified 
structure. The density of these electrons is a function of the height above the earth's surface and is dramatically affected by the effects of sunrise and sunset, especially at the lower altitudes. Also, the many parameters in the ionosphere are the function of the electron density. The ionosphere is conventionally divided into the D, E, and F-regions. The D-region lies between 60 and $95 \mathrm{~km}$, the E-region between 95 and $150 \mathrm{~km}$, and the Fregion lies above $150 \mathrm{~km}$. During daylight, it is possible to distinguish two separate layers within the F-region, the F1 (lower) and the F2 (upper) layers. During nighttime, these two layers combine into one single layer. The combined effect of gravitationally decreasing densities of neutral atoms and molecules and increasing intensity of ionizing solar ultraviolet radiation with increasing altitudes, gives a maximum plasma density during daytime in the F-region at a few hundred kilometers altitude. During daytime, the ratio of charged particles to neutral particles concentration can vary from $10^{-8}$ at $100 \mathrm{~km}$ to $10^{-4}$ at $300 \mathrm{~km}$ and $10^{-1}$ at $1000 \mathrm{~km}$ altitude. The main property of F-region consists of the free electrons.

As known, the permittivity and permeability parameters are related to electric and magnetic susceptibilities of material, on account of medium and moreover, the speed of electromagnetic wave and the characteristic impedance depend on any medium and the refractive index of medium gives detail information about any medium. Because of all of these reasons, $\varepsilon$ is a measure of refractive index, reflection, volume and wave polarization of electromagnetic, impedance of medium.

Propagation of electromagnetic waves in the atmosphere is influenced by the spatial distribution of the refractive index of the ionosphere [3]. The theory of ionospheric conductivity was developed by many scientists and is now quite well understood, though refinements are still made from time to time. The ionosphere carries electric currents because winds and electric fields drive ions and electrons. The direction of the drift is at right angles to the geomagnetic field [4]. Furthermore, electrical conductivity is an important central concept in space science, because it determines how driving forces, such as electric fields and thermosphere winds, couple to plasma motions and the resulting electric currents. The tensor of electrical conductivity finds application in all the areas of ionospheric electrodynamics and at all the latitudes [5]. On the other hand, the most important parameter determining the behavior of any medium is the dielectric constant, which at any frequency determines the refractive index, the form of wave in medium, to be polarized, the state of wave energy and the propagation of wave.

In this chapter, the behavior of electromagnetic waves emitted from within the ionospheric plasma and the analytical solutions are necessary to understand the characteristics of the environment will be defined. Problems in plasma physics at the conductivity, dielectric constants and refractive index will be defined according to the media parameters. When these expressions, using Maxwell's equations expressed in the wave dispersion equation, wave propagation, depending on the parameters of the environment will be examined. These statements are expressed in terms of Maxwell's equations using the wave dispersion equation, wave propagation, depending on the parameters of the environment will be examined. By examining of the dispersion relation, the types of wave occurred in the media and relaxation mechanisms, polarizations and conflicts caused by ionospheric amplitude attenuation of these waves will be obtained analytically. Thus, resolving problems of ionospheric plasma in the emitted radio waves, the basic information that will be understood. 


\section{Conductivity for ionospheric plasma}

One of the main parameters affecting the progression of the electromagnetic wave in a medium is the conductivity of the media. Conductivity of the media statement is obtained from motion equation of the charged particle that is taken account in the total force acting on charged particle in ionospheric plasma. Accordingly, the forces acting on charged particle is given as follows [6].

$$
\begin{aligned}
\text { Mass xacceleration } & =\text { Electrical forces } \\
& + \text { Magnetic forces } \\
& + \text { Shooting (gravitational) forces } \\
& + \text { Pressure changing forces } \\
& + \text { Collision forces }
\end{aligned}
$$

The plasma approach, which the thermal motion due to temperatures of particles, are neglected, is called cold plasma. In this study, because of the cold plasma approximation, any force does not effect on charged particles due to the temperature and therefore the term of pressure changes is ignored [7]. Likewise, the gravitational force from the gravity is negligible due to so small according to the electric and magnetic forces. In addition, due to the electron mass $\left(\mathrm{m}_{\mathrm{e}}\right)$ is very small according to the mass of the ion $\left(\mathrm{m}_{\mathrm{i}}\right)\left(\mathrm{m}_{\mathrm{e}}<<\mathrm{m}_{\mathrm{i}}\right)$ and thus the electron collision frequency is greater than the ion collision frequency, the movement of ions in the plasma is ignored near the electron movement. Accordingly, Equation (1) is written as the following equation for the electron:

$$
\mathrm{m}_{\mathrm{e}} \frac{\mathrm{d} \mathbf{V}_{\mathrm{e}}}{\mathrm{dt}}=-\mathrm{e}\left(\mathbf{E}+\mathbf{V}_{\mathrm{e}} \times \mathbf{B}\right)-\mathrm{m}_{\mathrm{e}} \mathrm{v}_{\mathrm{e}} \mathbf{V}_{\mathrm{e}}
$$

This expression is called the Langevin equation [8]. Where, the electron collision frequency $v_{\mathrm{e}}$ is sum of the electron-ion collision frequency $\left(v_{\mathrm{ei}}\right)$ and electron-neutral particle collision $\left(v_{\text {en }}\right)$ frequency.

\subsection{DC conductivity}

The first application of the Langevin equation is steady flow caused by of the electric field. For this, $\dot{\mathbf{V}}_{\mathrm{e}}=0$. Accordingly, the Langevin equation for electrons can be defined as follows:

$$
0=-\mathrm{e}\left(\mathbf{E}+\mathbf{V}_{\mathrm{e}} \times \mathbf{B}\right)-\mathrm{m}_{\mathrm{e}} v_{\mathrm{e}} \mathbf{V}_{\mathrm{e}}
$$

Taking into consideration that the current density is $\mathbf{J}_{\mathrm{e}}=-\mathrm{eN}_{\mathrm{e}} \mathbf{V}_{\mathrm{e}}$, in order to obtained the current density, both sides of the expression is multiplied by the term of $-\mathrm{eN}_{\mathrm{e}}$. In this case,

$$
\mathrm{e}^{2} \mathrm{~N}_{\mathrm{e}}\left(\mathbf{E}+\mathbf{V}_{\mathrm{e}} \times \mathbf{B}\right)=-\mathrm{m}_{\mathrm{e}} \mathrm{eN} \mathrm{N}_{\mathrm{e}} \boldsymbol{V}_{\mathrm{e}} \mathbf{V}_{\mathrm{e}}
$$

and the expression is transformed as follows:

$$
\mathrm{e}^{2} \mathrm{~N}_{\mathrm{e}}\left(\mathbf{E}+\mathbf{V}_{\mathrm{e}} \times \mathbf{B}\right)=\mathrm{m}_{\mathrm{e}} v_{\mathrm{e}} \mathbf{J}
$$


For the current density, the following equation is obtained:

$$
\mathbf{J}=\frac{\mathrm{e}^{2} \mathrm{~N}_{\mathrm{e}}}{\mathrm{m}_{\mathrm{e}} \mathrm{v}_{\mathrm{e}}}\left(\mathbf{E}+\mathbf{V}_{\mathrm{e}} \times \mathbf{B}\right)
$$

Taking into consideration that $\mathbf{J}=\sigma \cdot \mathbf{E}$, the generalized Ohm Law can be defined by,

$$
\mathbf{J}=\sigma_{0}\left(\mathbf{E}+\mathbf{V}_{\mathrm{e}} \times \mathbf{B}\right)
$$

Where, $\sigma_{0}=\frac{\mathrm{e}^{2} \mathrm{~N}_{\mathrm{e}}}{\mathrm{m}_{\mathrm{e}} v_{\mathrm{e}}}$ is the dc conductivity of te plasma. If $v_{\mathrm{e}} \rightarrow 0$, the term $\sigma_{0}$ is being $\sigma_{0} \rightarrow \infty$. In this case, if the $\mathbf{E}+\mathbf{V}_{\mathrm{e}} \times \mathbf{B}=0$, the current is finite. For this reason, the case of $\sigma_{0}=\infty$, the state $\mathbf{E}+\mathbf{V}_{\mathrm{e}} \times \mathbf{B}=0$ is called OHM law for the plasma. The geometry of the velocity and electric field and magnetic field is as shown in Figure 1, namely $\mathbf{V}=\hat{\mathrm{x}} \mathrm{V}_{\mathrm{x}}+\hat{\mathrm{y}} \mathrm{V}_{\mathrm{y}}+\hat{\mathrm{z}} \mathrm{V}_{\mathrm{z}}, \mathbf{E}=\hat{\mathrm{x}} \mathrm{E}_{\mathrm{x}}+\hat{\mathrm{y}} \mathrm{E}_{\mathrm{y}}+\hat{\mathrm{z}} \mathrm{E}_{\mathrm{z}}$ and $\mathbf{B}=\hat{\mathrm{z}} \mathrm{B}$, the $\mathbf{V}_{\mathrm{e}} \times \mathbf{B}$ term in Equation (7) is being as follows:

$$
\mathbf{V}_{\mathrm{e}} \times \mathbf{B}=\left|\begin{array}{ccc}
\hat{x} & \hat{y} & \hat{z} \\
\mathrm{~V}_{\mathrm{x}} & \mathrm{V}_{\mathrm{y}} & \mathrm{V}_{\mathrm{z}} \\
0 & 0 & \mathrm{~B}
\end{array}\right|=\hat{x}\left(\mathrm{~V}_{\mathrm{y}} \mathrm{B}\right)-\hat{\mathrm{y}}\left(\mathrm{V}_{\mathrm{x}} \mathrm{B}\right)
$$

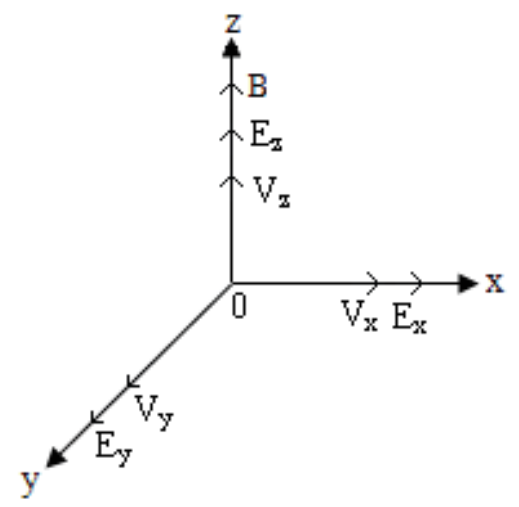

Fig. 1. The geometry of the velocity, electric field and magnetic field

Here, by the necessary mathematical operations are made; the following expressions are obtained for three components of current density:

$$
\begin{gathered}
J_{x}=\frac{e^{2} N_{e}}{m_{e} v_{e}}\left(E_{x}+V_{y} B\right)=\frac{e^{2} N_{e}}{m_{e} v_{e}} E_{x}+\frac{e^{2} N_{e} V_{y} B}{m_{e} v_{e}} \\
J_{y}=\frac{e^{2} N_{e}}{m_{e} v_{e}}\left(E_{y}-V_{x} B\right)=\frac{e^{2} N_{e}}{m_{e} v_{e}} E_{y}-\frac{e^{2} N_{e} V_{x} B}{m_{e} v_{e}}
\end{gathered}
$$




$$
J_{z}=\frac{e^{2} N_{e}}{m_{e} v_{e}} E_{z}=\sigma_{0} E_{z}
$$

Taking into consideration that cyclotron frequency $\omega_{\mathrm{ce}}=-\frac{\mathrm{eB}}{\mathrm{m}_{\mathrm{e}}}$ for the electron, if the second terms on the right side of this expression is written in terms of current density $\mathbf{J}$ and the necessary procedures are made, $\mathrm{x}$ and $\mathrm{y}$ components of current density are obtained as follows:

$$
\begin{aligned}
& J_{x}=\frac{e^{2} N_{e} v_{e}}{m_{e}\left(v_{e}^{2}+\omega_{c e}{ }^{2}\right)} E_{x}+\frac{e^{2} N_{e} \omega_{c e}}{m_{e}\left(v_{e}{ }^{2}+\omega_{c e}{ }^{2}\right)} E_{y} \\
& J_{y}=\frac{e^{2} N_{e} \omega_{c e}}{m_{e}\left(v_{e}{ }^{2}+\omega_{c e}{ }^{2}\right)} E_{x}+\frac{e^{2} N_{e} v_{e}}{m_{e}\left(v_{e}{ }^{2}+\omega_{c e}{ }^{2}\right)} E_{y}
\end{aligned}
$$

The expressions $J_{x}, J_{y}$ and $J_{z}$ in the tensor form of $([\mathbf{J}]=[\sigma] \cdot[\mathbf{E}])$ can be written as follows:

$$
\left[\begin{array}{c}
J_{\mathrm{x}} \\
\mathrm{J}_{\mathrm{y}} \\
\mathrm{J}_{\mathrm{z}}
\end{array}\right]=\left[\begin{array}{ccc}
\frac{\mathrm{e}^{2} \mathrm{~N}_{\mathrm{e}} v_{\mathrm{e}}}{\mathrm{m}_{\mathrm{e}}\left(v_{\mathrm{e}}^{2}+\omega_{\mathrm{ce}}{ }^{2}\right)} & \frac{\mathrm{e}^{2} \mathrm{~N}_{\mathrm{e}} \omega_{\mathrm{ce}}}{\mathrm{m}_{\mathrm{e}}\left(v_{\mathrm{e}}^{2}+\omega_{\mathrm{ce}}{ }^{2}\right)} & 0 \\
-\frac{\mathrm{e}^{2} \mathrm{~N}_{\mathrm{e}} \omega_{\mathrm{ce}}}{\mathrm{m}_{\mathrm{e}}\left(v_{\mathrm{e}}{ }^{2}+\omega_{\mathrm{ce}}{ }^{2}\right)} & \frac{\mathrm{e}^{2} \mathrm{~N}_{\mathrm{e}} v_{\mathrm{e}}}{\mathrm{m}_{\mathrm{e}}\left(v_{\mathrm{e}}{ }^{2}+\omega_{\mathrm{ce}}{ }^{2}\right)} & 0 \\
0 & 0 & \frac{\mathrm{e}^{2} \mathrm{~N}_{\mathrm{e}}}{\mathrm{m}_{\mathrm{e}} v_{\mathrm{e}}}
\end{array}\right] \cdot\left[\begin{array}{c}
\mathrm{E}_{\mathrm{x}} \\
\mathrm{E}_{\mathrm{y}} \\
\mathrm{E}_{\mathrm{z}}
\end{array}\right]
$$

Here, the conductivity tensor is called as dc conductivity tensor. As can be seen in this expression, $\sigma_{0}=\frac{\mathrm{e}^{2} \mathrm{~N}_{\mathrm{e}}}{\mathrm{m}_{\mathrm{e}} v_{\mathrm{e}}}$ term provides the flow of the current in $\mathbf{B}$ direction. If the conductivity tensor is defined as follows:

$$
\sigma=\left[\begin{array}{ccc}
\sigma_{1} & \sigma_{2} & 0 \\
-\sigma_{2} & \sigma_{1} & 0 \\
0 & 0 & \sigma_{0}
\end{array}\right]
$$

$\sigma_{1}\left(=\frac{\mathrm{e}^{2} \mathrm{~N}_{\mathrm{e}} v_{\mathrm{e}}}{\mathrm{m}_{\mathrm{e}}\left(v_{\mathrm{e}}^{2}+\omega_{\mathrm{ce}}^{2}\right)}\right)$ Conductivity is called as perpendicular conductivity $\left(\sigma_{\perp}\right)$. When the $\mathbf{E}$ is perpendicular to the $\mathbf{B}$, this conductivity provides to flow the current in the direction of $\mathbf{E}$. The conductivity defined by $\sigma_{2}\left(=\frac{\mathrm{e}^{2} \mathrm{~N}_{\mathrm{e}} \omega_{\mathrm{ce}}}{\mathrm{m}_{\mathrm{e}}\left(\nu_{\mathrm{e}}{ }^{2}+\omega_{\mathrm{ce}}{ }^{2}\right)}\right)$ is called as Hall conductivity $\left(\sigma_{\mathrm{H}}\right)$ and it provides the current, which is perpendicular to the both of the $\mathbf{E}$ and $\mathbf{B}$ (Figure 2). 


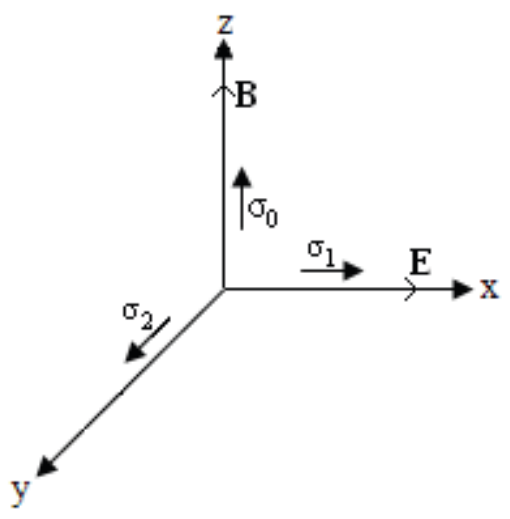

Fig. 2. The direction of the currents provided by the conductivities $\sigma_{0}, \sigma_{1}$ and $\sigma_{2}$ according to the geometry of $\mathbf{E}$ and $\mathbf{B}$

If the $\sigma_{1}=\frac{\sigma_{0} v_{\mathrm{e}}^{2}}{v_{\mathrm{e}}{ }^{2}+\omega_{\mathrm{ce}}{ }^{2}}=\frac{\sigma_{0}}{1+\frac{\omega_{\mathrm{ce}}{ }^{2}}{v_{\mathrm{e}}{ }^{2}}}$ and $\sigma_{2}=\frac{\sigma_{0} v_{\mathrm{e}} \omega_{\mathrm{ce}}}{v_{\mathrm{e}}{ }^{2}+\omega_{\mathrm{ce}}{ }^{2}}=\frac{\sigma_{0} \frac{\omega_{\mathrm{ce}}}{v_{\mathrm{e}}}}{1+\frac{\omega_{\mathrm{ce}}{ }^{2}}{v_{\mathrm{e}}{ }^{2}}}$, the variation of the conductivities is being as shown in Figure 3. As shown in Figure 3, after the $\frac{\omega_{\mathrm{ce}}}{v_{\mathrm{e}}}>1 \sigma_{1}$ term decreases rapidly. Thus, the current perpendicular to the magnetic field is very small [8].

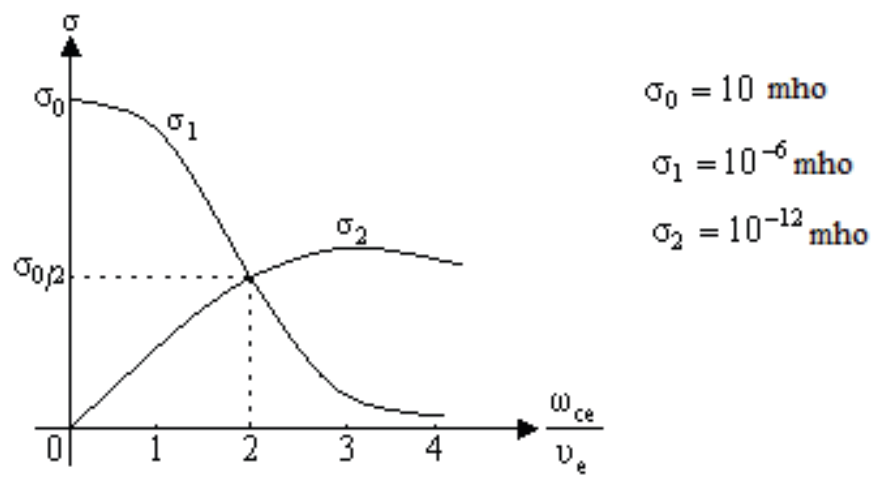

Fig. 3. The variation of $\sigma_{1}$ and $\sigma_{2}$ conductivities

\subsection{AC conductivity}

There is no steady-state in the ac conductivity. Taking into consideration that the fields vary as $\mathrm{e}^{-\mathrm{i} \omega \mathrm{t}}$, the Equation (2) is transformed as follows:

$$
-\mathrm{m}_{\mathrm{e}} \mathrm{i} \omega \mathbf{V}_{\mathrm{e}}=-\mathrm{e}\left(\mathbf{E}+\mathbf{V}_{\mathbf{e}} \times \mathbf{B}\right)-\mathrm{m}_{\mathrm{e}} v_{\mathrm{e}} \mathbf{V}_{\mathbf{e}}
$$


According to the geometry given in Figure 1, this expression in terms of current density can be defined as follows:

$$
\mathbf{J}\left(v_{\mathrm{e}}-\mathrm{i} \omega\right)=\frac{\mathrm{e}^{2} \mathrm{~N}_{\mathrm{e}}}{\mathrm{m}_{\mathrm{e}}} \mathbf{E}-\frac{\mathrm{e}^{2} \mathrm{~N}_{\mathrm{e}}}{\mathrm{m}_{\mathrm{e}}}\left(\mathbf{V}_{\mathrm{e}} \times \mathbf{B}\right)
$$

Thus, current density components are obtained as follows:

$$
\begin{gathered}
J_{x}=\frac{e^{2} N_{e}}{m_{e}\left(v_{e}-i \omega\right)} E_{x}+\frac{e^{2} N_{e}}{m_{e}\left(v_{e}-i \omega\right)} V_{y} B \\
J_{y}=\frac{e^{2} N_{e}}{m_{e}\left(v_{e}-i \omega\right)} E_{y}-\frac{e^{2} N_{e}}{m_{e}\left(v_{e}-i \omega\right)} V_{x} B \\
J_{z}=\frac{e^{2} N_{e}}{m_{e}\left(v_{e}-i \omega\right)} E_{z}
\end{gathered}
$$

Considering the $\omega_{\mathrm{ce}}$ cyclotron frequency, the components $\mathrm{J}_{\mathrm{x}}$ and $\mathrm{J}_{\mathrm{y}}$ can be obtained as follows:

$$
\begin{gathered}
J_{x}=\frac{e^{2} N_{e}\left(v_{e}-i \omega\right)}{m_{e}\left[\omega_{c e}^{2}+\left(v_{e}-i \omega\right)^{2}\right]} E_{x}+\frac{e^{2} N_{e} \omega_{c e}}{m_{e}\left[\omega_{c e}^{2}+\left(v_{e}-i \omega\right)^{2}\right]} E_{y} \\
J_{y}=-\frac{e^{2} N_{e} \omega_{c e}}{m_{e}\left[\omega_{c e}^{2}+\left(v_{e}-i \omega\right)^{2}\right]} E_{x}+\frac{e^{2} N_{e}\left(v_{e}-i \omega\right)}{m_{e}\left[\omega_{c e}^{2}+\left(v_{e}-i \omega\right)^{2}\right]} E_{y}
\end{gathered}
$$

If the expressions (20)-(22) in the tensor form and the conductivity tensor $\sigma^{\prime}$ are defined as follows,

$$
\begin{gathered}
{\left[\begin{array}{l}
\mathrm{J}_{\mathrm{x}} \\
\mathrm{J}_{\mathrm{y}} \\
\mathrm{J}_{\mathrm{z}}
\end{array}\right]=\left[\begin{array}{ccc}
\sigma_{1}^{\prime} & \sigma_{2}^{\prime} & 0 \\
\sigma_{2}^{\prime} & \sigma_{1}^{\prime} & 0 \\
0 & 0 & \sigma_{0}^{\prime}
\end{array}\right] \cdot\left[\begin{array}{l}
\mathrm{E}_{\mathrm{x}} \\
\mathrm{E}_{\mathrm{y}} \\
\mathrm{E}_{\mathrm{z}}
\end{array}\right]} \\
\sigma^{\prime}=\left[\begin{array}{ccc}
\sigma_{1}^{\prime} & \sigma_{2}^{\prime} & 0 \\
-\sigma_{2}^{\prime} & \sigma_{1}^{\prime} & 0 \\
0 & 0 & \sigma_{0}^{\prime}
\end{array}\right]
\end{gathered}
$$

The conductivities $\sigma_{0}^{\prime}, \sigma_{1}^{\prime}$ and $\sigma_{2}^{\prime}$ will be $\sigma_{0}^{\prime}=\frac{\mathrm{e}^{2} \mathrm{~N}_{\mathrm{e}}}{\mathrm{m}_{\mathrm{e}}\left(v_{\mathrm{e}}-\mathrm{i} \omega\right)}, \sigma_{1}^{\prime}=\frac{\mathrm{e}^{2} \mathrm{~N}_{\mathrm{e}}\left(v_{\mathrm{e}}-\mathrm{i} \omega\right)}{\mathrm{m}_{\mathrm{e}}\left[\omega_{\mathrm{ce}}{ }^{2}+\left(v_{\mathrm{e}}-\mathrm{i} \omega\right)^{2}\right]^{2}}$, $\sigma_{2}^{\prime}=\frac{\mathrm{e}^{2} \mathrm{~N}_{\mathrm{e}} \omega_{\mathrm{ce}}}{\mathrm{m}_{\mathrm{e}}\left[\omega_{\mathrm{ce}}^{2}+\left(v_{\mathrm{e}}-\mathrm{i} \omega\right)^{2}\right]}$. Considering the oscillations frequency of the plasma for the 
electron as $\omega_{\mathrm{pe}}^{2}\left(=\frac{\mathrm{e}^{2} \mathrm{~N}_{\mathrm{e}}}{\mathrm{m}_{\mathrm{e}} \varepsilon_{0}}\right)$, these equations can be written as $\sigma_{0}^{\prime}=\frac{\omega_{\mathrm{pe}}^{2} \varepsilon_{0}}{\left(v_{\mathrm{e}}-\mathrm{i} \omega\right)}$, $\sigma_{1}^{\prime}=\frac{\omega_{\mathrm{pe}}^{2} \varepsilon_{0}\left(v_{\mathrm{e}}-\mathrm{i} \omega\right)}{\left[\omega_{\mathrm{ce}}^{2}+\left(v_{\mathrm{e}}-\mathrm{i} \omega\right)^{2}\right]}, \sigma_{2}^{\prime}=\frac{\omega_{\mathrm{pe}}^{2} \varepsilon_{0} \omega_{\mathrm{ce}}}{\left[\omega_{\mathrm{ce}}^{2}+\left(v_{\mathrm{e}}-\mathrm{i} \omega\right)^{2}\right]}$, respectively. Where, as in dc conductivity $\sigma_{0}^{\prime}$ is the conductivity of the magnetic field direction, $\sigma_{1}^{\prime}$ is the conductivity perpendicular to the magnetic field and $\sigma_{2}^{\prime}$ is the conductivity perpendicular to both of the electrical and the magnetic fields, respectively. If $\sigma_{0}^{\prime}>\sigma_{1}^{\prime}>\sigma_{2}^{\prime}$ and $\left(v_{\mathrm{e}}-\mathrm{i} \omega\right)$, the ac conductivity tensor transforms to the dc conductivity tensor.

Example: ac conductivity tensor according to the geometry given in Figure 4 is obtained as in Equation (25):

$$
\sigma^{\prime}=\left[\begin{array}{ccc}
\frac{\omega_{\mathrm{pe}}{ }^{2} \varepsilon_{0}\left(v_{\mathrm{e}}-\mathrm{i} \omega\right)}{\left[\omega_{\mathrm{ce}}{ }^{2}+\left(v_{\mathrm{e}}-i \omega\right)^{2}\right]} & 0 & -\frac{\omega_{\mathrm{pe}}{ }^{2} \varepsilon_{0} \omega_{\mathrm{ce}}}{\left[\omega_{\mathrm{ce}}{ }^{2}+\left(v_{\mathrm{e}}-i \omega\right)^{2}\right]} \\
0 & \frac{\omega_{\mathrm{pe}}^{2} \varepsilon_{0}}{\left(v_{\mathrm{e}}-i \omega\right)} & 0 \\
\frac{\omega_{\mathrm{pe}}{ }^{2} \varepsilon_{0} \omega_{\mathrm{ce}}}{\left[\omega_{\mathrm{ce}}{ }^{2}+\left(v_{\mathrm{e}}-i \omega\right)^{2}\right]} & 0 & \frac{\omega_{\mathrm{pe}}^{2} \varepsilon_{0}\left(v_{\mathrm{e}}-i \omega\right)}{\left[\omega_{\mathrm{ce}}{ }^{2}+\left(v_{\mathrm{e}}-i \omega\right)^{2}\right]}
\end{array}\right]
$$

If the magnetic field is only $\mathbf{B}=\hat{\mathrm{x}} \mathrm{B}$ direction, ac conductivity tensor becomes as follows:

$$
\sigma^{\prime}=\left[\begin{array}{ccc}
\frac{\omega_{\mathrm{pe}}{ }^{2} \varepsilon_{0}}{\left(v_{\mathrm{e}}-\mathrm{i} \omega\right)} & 0 & 0 \\
0 & \frac{\omega_{\mathrm{pe}}{ }^{2} \varepsilon_{0}\left(v_{\mathrm{e}}-\mathrm{i} \omega\right)}{\left[\omega_{\mathrm{ce}}{ }^{2}+\left(v_{\mathrm{e}}-\mathrm{i} \omega\right)^{2}\right]} & \frac{\omega_{\mathrm{pe}}{ }^{2} \varepsilon_{0} \omega_{\mathrm{ce}}}{\left[\omega_{\mathrm{ce}}{ }^{2}+\left(v_{\mathrm{e}}-i \omega\right)^{2}\right]} \\
0 & -\frac{\omega_{\mathrm{pe}}{ }^{2} \varepsilon_{0} \omega_{\mathrm{ce}}}{\left[\omega_{\mathrm{ce}}{ }^{2}+\left(v_{\mathrm{e}}-i \omega\right)^{2}\right]} & \frac{\omega_{\mathrm{pe}}{ }^{2} \varepsilon_{0}\left(v_{\mathrm{e}}-i \omega\right)}{\left[\omega_{\mathrm{ce}}{ }^{2}+\left(v_{\mathrm{e}}-i \omega\right)^{2}\right]}
\end{array}\right]
$$

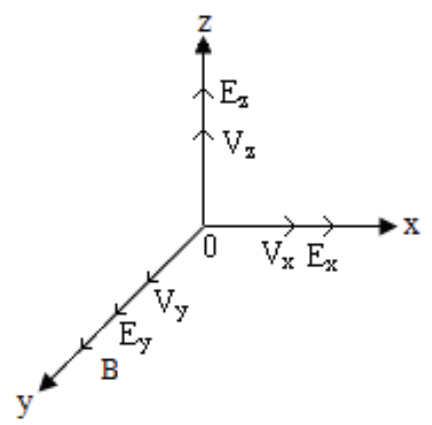

Fig. 4. The geometry of the velocity and electric field and magnetic field 
Example: If the magnetic field has two-dimensional geometry as given in Figure 5 $\left(\mathbf{B}=\hat{\mathrm{y}} \mathrm{B}_{\mathrm{y}}+\hat{\mathrm{z}} \mathrm{B}_{\mathrm{z}}=\hat{\mathrm{y}} \mathrm{B} \operatorname{Cos} \theta+\hat{z} \mathrm{BSin} \theta\right)$, the conductivity tensor are defined as in Equation (27):

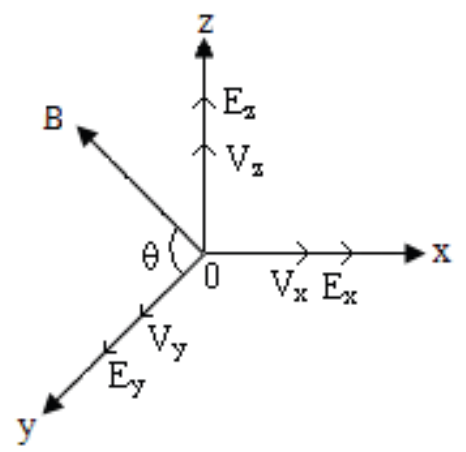

Fig. 5. The geometry of the velocity and electric field and magnetic field

$$
\sigma^{\prime}=\left[\begin{array}{ccc}
\sigma_{1}^{\prime} & \sigma_{2}^{\prime} \operatorname{Sin} \theta & -\sigma_{2}^{\prime} \operatorname{Cos} \theta \\
\sigma_{2}^{\prime} \operatorname{Sin} \theta & \sigma_{0}^{\prime}+\left(\sigma_{0}^{\prime}-\sigma_{1}^{\prime}\right) \operatorname{Sin}^{2} \theta & -\left(\sigma_{0}^{\prime}-\sigma_{1}^{\prime}\right) \operatorname{Cos} \theta \operatorname{Sin} \theta \\
\sigma_{2}^{\prime} \operatorname{Cos} \theta & \left(\sigma_{0}^{\prime}-\sigma_{1}^{\prime}\right) \operatorname{Cos} \theta \operatorname{Sin} \theta & \sigma_{0}^{\prime}+\left(\sigma_{0}^{\prime}-\sigma_{1}^{\prime}\right) \operatorname{Cos}^{2} \theta
\end{array}\right]
$$

\subsection{Earth's magnetic field and ionospheric conductivity}

In this section, the Langevin equation defined by Equation (16) for ac conductivity will be discussed. However, the true magnetic field in the Earth's northern half-sphere given in Figure 6 will be dealt with. Accordingly, in the selected Cartesian coordinate system, the $x$ axis represents the geographic east, the y-axis represents the the geographic North and z-axis represents the up in the vertical direction, the magnetic field can be defined as follows [9]:

$$
\mathbf{B}=\hat{\mathrm{x}} \mathrm{BC} \operatorname{Cos} \operatorname{Sin} \mathrm{D}+\hat{\mathrm{y}} \mathrm{BCosICos} \mathrm{D}-\hat{z} \mathrm{BSinI}
$$

Where, I is the dip angle and $\mathrm{D}$ is the declination angle (between the magnetic north and the geographic north).

Thus, the term of $\mathbf{V}_{\mathrm{e}} \times \mathbf{B}$ in Equation (16) can be obtained as in Equation (29).

$$
\mathbf{V}_{\mathrm{e}} \times \mathbf{B}=\left|\begin{array}{ccc}
\hat{\mathrm{x}} & \hat{\mathrm{y}} & \hat{\mathrm{z}} \\
\mathrm{V}_{\mathrm{x}} & \mathrm{V}_{\mathrm{y}} & \mathrm{V}_{\mathrm{z}} \\
\mathrm{BCosISinD} & \mathrm{BCosICosD} & -\mathrm{BSinI}
\end{array}\right|=\begin{aligned}
& \hat{\mathrm{x}}\left(-\mathrm{V}_{\mathrm{y}} \mathrm{BSinI}-\mathrm{V}_{\mathrm{z}} \mathrm{BC} \operatorname{Cos} \operatorname{Cos} \mathrm{D}\right) \\
& \\
&
\end{aligned}
$$

If this expression is written in Equation (16) and the necessary mathematical manipulations are made, three equations are obtained for $\mathrm{x}, \mathrm{y}$ and $\mathrm{z}$ directions as follows:

$$
-\frac{\mathrm{e}}{\mathrm{m}_{\mathrm{e}}\left(v_{\mathrm{e}}-\mathrm{i} \omega\right)} \mathrm{E}_{\mathrm{x}}=\mathrm{V}_{\mathrm{x}}+\frac{\omega_{\mathrm{ce}} \operatorname{SinI}}{\left(v_{\mathrm{e}}-\mathrm{i} \omega\right)} \mathrm{V}_{\mathrm{y}}+\frac{\omega_{\mathrm{ce}} \operatorname{Cos} I \operatorname{Cos} \mathrm{D}}{\left(v_{\mathrm{e}}-\mathrm{i} \omega\right)} \mathrm{V}_{\mathrm{z}}
$$




$$
\begin{gathered}
-\frac{e}{m_{e}\left(v_{e}-i \omega\right)} E_{y}=V_{y}-\frac{\omega_{c e} \operatorname{SinI}}{\left(v_{e}-i \omega\right)} V_{x}-\frac{\omega_{c e} \operatorname{CosISinD}}{\left(v_{e}-i \omega\right)} V_{z} \\
-\frac{e}{m_{e}\left(v_{e}-i \omega\right)} E_{z}=V_{z}-\frac{\omega_{c e} \operatorname{Cos} I \operatorname{Cos} D}{\left(v_{e}-i \omega\right)} V_{x}+\frac{\omega_{c e} \operatorname{CosISinD}}{\left(v_{e}-i \omega\right)} V_{y}
\end{gathered}
$$
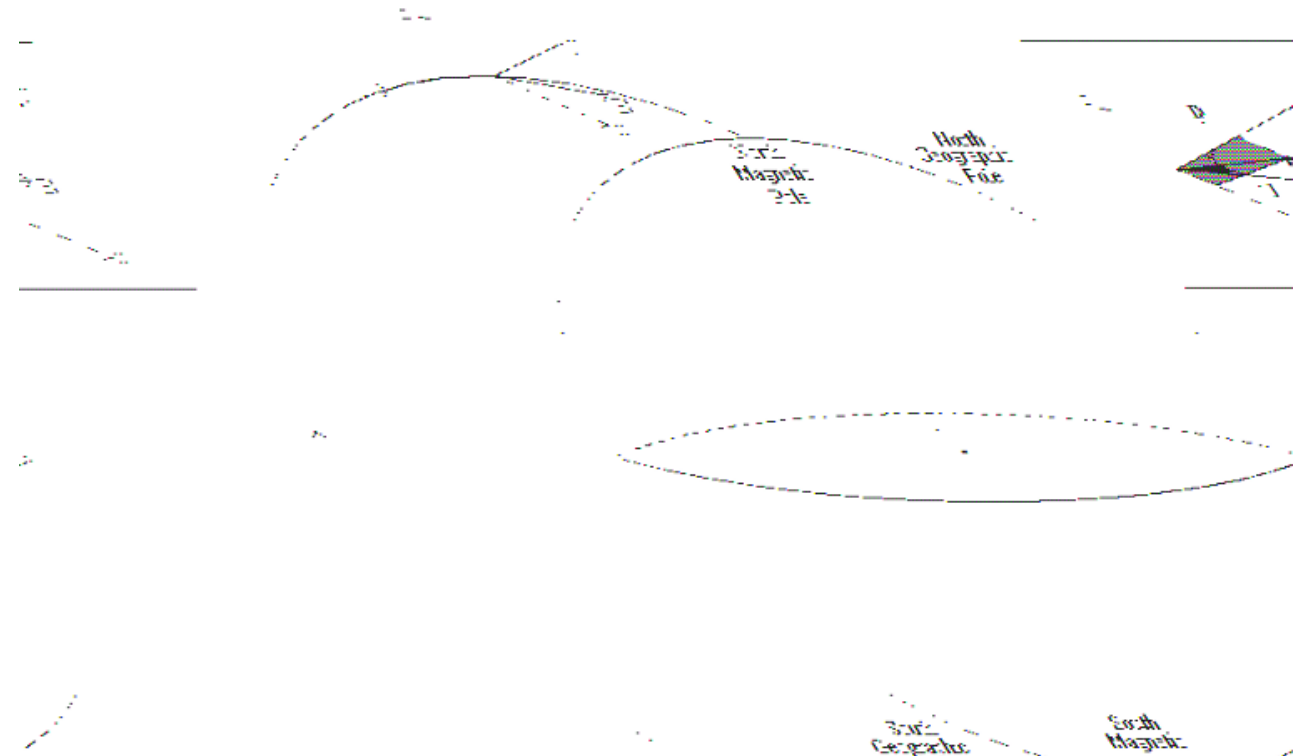

Fig. 6. Earth's magnetic field on north hemisphere

In order to obtain the current density, if the both sides of the expression is multiplied by $-\mathrm{eN}_{\mathrm{e}}$, the Equations (30)-(32) transform to Equations (33)-(35).

$$
\begin{gathered}
\frac{\mathrm{e}^{2} N_{e}}{\mathrm{~m}_{\mathrm{e}}\left(v_{\mathrm{e}}-\mathrm{i} \omega\right)} \mathrm{E}_{\mathrm{x}}=\mathrm{J}_{\mathrm{x}}+\frac{\omega_{\mathrm{ce}} \operatorname{SinI}}{\left(v_{\mathrm{e}}-\mathrm{i} \omega\right)} \mathrm{J}_{\mathrm{y}}+\frac{\omega_{\mathrm{ce}} \operatorname{Cos} I \operatorname{Cos} D}{\left(v_{\mathrm{e}}-\mathrm{i} \omega\right)} \mathrm{J}_{\mathrm{z}} \\
\frac{\mathrm{e}^{2} \mathrm{~N}_{\mathrm{e}}}{\mathrm{m}_{\mathrm{e}}\left(v_{\mathrm{e}}-\mathrm{i} \omega\right)} \mathrm{E}_{\mathrm{y}}=-\frac{\omega_{\mathrm{ce}} \operatorname{SinI}}{\left(v_{\mathrm{e}}-\mathrm{i} \omega\right)} \mathrm{J}_{\mathrm{x}}+\mathrm{J}_{\mathrm{y}}-\frac{\omega_{\mathrm{ce}} \operatorname{CosISinD}}{\left(v_{\mathrm{e}}-\mathrm{i} \omega\right)} \mathrm{J}_{\mathrm{z}} \\
\frac{\mathrm{e}^{2} \mathrm{~N}_{\mathrm{e}}}{\mathrm{m}_{\mathrm{e}}\left(v_{\mathrm{e}}-\mathrm{i} \omega\right)} \mathrm{E}_{\mathrm{z}}=-\frac{\omega_{\mathrm{ce}} \operatorname{Cos} \operatorname{Cos} \mathrm{D}}{\left(v_{\mathrm{e}}-\mathrm{i} \omega\right)} \mathrm{J}_{\mathrm{x}}+\frac{\omega_{\mathrm{ce}} \operatorname{Cos} I \operatorname{Sin} D}{\left(v_{\mathrm{e}}-\mathrm{i} \omega\right)} \mathrm{J}_{\mathrm{y}}+\mathrm{J}_{\mathrm{z}}
\end{gathered}
$$

These equations are first order linear equation in three unknowns. It is impossible to obtain this expression in the solution of each other. Therefore, whether or not the solution, it is necessary to express by using, "Cramer's Method". This method gives the solution of linear 
equation system which has coefficients matrix as square matrix. According to this method, if the determinant of the coefficients matrix of the system is non-zero, the equation has a single solution. Let accept the $\frac{e^{2} N_{e}}{m_{e}\left(v_{e}-i \omega\right)}=\sigma_{0}^{\prime}, \quad \frac{\omega_{c e} \operatorname{SinI}}{\left(v_{e}-i \omega\right)}=a, \quad \frac{\omega_{c e} \operatorname{Cos} I \operatorname{Cos} D}{\left(v_{e}-i \omega\right)}=b \quad$ and $\frac{\omega_{\text {ce }} \operatorname{CosISinD}}{\left(v_{\mathrm{e}}-\mathrm{i} \omega\right)}=\mathrm{c}$ in Equations (33)-(35). Thus, these tree equations can be defined as follows:

$$
\left[\begin{array}{c}
\sigma_{0}^{\prime} \mathrm{E}_{\mathrm{x}} \\
\sigma_{0}^{\prime} \mathrm{E}_{\mathrm{y}} \\
\sigma_{0}^{\prime} \mathrm{E}_{\mathrm{z}}
\end{array}\right]=\left[\begin{array}{rcc}
1 & \mathrm{a} & \mathrm{b} \\
-\mathrm{a} & 1 & -\mathrm{c} \\
-\mathrm{b} & \mathrm{c} & 1
\end{array}\right] \cdot\left[\begin{array}{c}
\mathrm{J}_{\mathrm{x}} \\
\mathrm{J}_{\mathrm{y}} \\
\mathrm{J}_{\mathrm{z}}
\end{array}\right]
$$

Here, the coefficients matrix is named as A, by taking the determinant of this matrix, the Equation (37) can be obtained.

$$
\operatorname{det} A=\left|\begin{array}{ccc}
1 & a & b \\
-a & 1 & -c \\
-b & c & 1
\end{array}\right|=1+a^{2}+b^{2}+c^{2} \neq 0
$$

This tells us that one solution of the equation. According to Cramer solution is as follows:

$$
\mathrm{J}_{\mathrm{x}}=\frac{\operatorname{det} \mathrm{A}_{1}}{\operatorname{det} \mathrm{A}}, \quad \mathrm{J}_{\mathrm{y}}=\frac{\operatorname{det} \mathrm{A}_{2}}{\operatorname{det} \mathrm{A}}, \quad \mathrm{J}_{\mathrm{z}}=\frac{\operatorname{det} \mathrm{A}_{3}}{\operatorname{det} \mathrm{A}}
$$

Here, the terms of $A_{1}, A_{2}$ and $A_{3}$ are defined as follows:

$$
\operatorname{det} \mathrm{A}_{1}=\left|\begin{array}{ccc}
\sigma_{0}^{\prime} \mathrm{E}_{\mathrm{x}} & \mathrm{a} & \mathrm{b} \\
\sigma_{0}^{\prime} \mathrm{E}_{\mathrm{y}} & 1 & -\mathrm{c} \\
\sigma_{0}^{\prime} \mathrm{E}_{\mathrm{z}} & \mathrm{c} & 1
\end{array}\right|, \quad \operatorname{det} \mathrm{A}_{2}=\left|\begin{array}{ccc}
1 & \sigma_{0}^{\prime} \mathrm{E}_{\mathrm{x}} & \mathrm{b} \\
-\mathrm{a} & \sigma_{0}^{\prime} \mathrm{E}_{\mathrm{y}} & -\mathrm{c} \\
-\mathrm{b} & \sigma_{0}^{\prime} \mathrm{E}_{\mathrm{z}} & 1
\end{array}\right|, \quad \operatorname{det} \mathrm{A}_{3}=\left|\begin{array}{ccc}
1 & \mathrm{a} & \sigma_{0}^{\prime} \mathrm{E}_{\mathrm{x}} \\
-\mathrm{a} & 1 & \sigma_{0}^{\prime} \mathrm{E}_{\mathrm{y}} \\
-\mathrm{b} & \mathrm{c} & \sigma_{0}^{\prime} \mathrm{E}_{\mathrm{z}}
\end{array}\right|
$$

In this statement, the $\mathrm{x}$ direction current density is resolved as in Equation (40).

$$
J_{x}=\frac{\operatorname{det} A_{1}}{\operatorname{det} A}=\frac{\sigma_{0}^{\prime}\left(1+c^{2}\right)}{1+a^{2}+b^{2}+c^{2}} E_{x}+\frac{\sigma_{0}^{\prime}(b c-a)}{1+a^{2}+b^{2}+c^{2}} E_{y}+\frac{\sigma_{0}^{\prime}(-a c-b)}{1+a^{2}+b^{2}+c^{2}} E_{z}
$$

For the $a, b$ and $c$, if the instead of the expressions given above are written, the $J_{x}$ term is obtained as follows:

$$
\begin{aligned}
\mathrm{J}_{\mathrm{x}} & =\frac{\sigma_{0}^{\prime}\left[\left(v_{\mathrm{e}}-\mathrm{i} \omega\right)^{2}+\omega_{\mathrm{ce}}^{2} \operatorname{Cos}^{2} \operatorname{ISin}^{2} \mathrm{D}\right]}{\left[\left(\mathrm{v}_{\mathrm{e}}-\mathrm{i} \omega\right)^{2}+\omega_{\mathrm{ce}}^{2}\right]} \mathrm{E}_{\mathrm{x}}+\frac{\sigma_{0}^{\prime}\left[\omega_{\mathrm{ce}}^{2} \operatorname{Cos}^{2} \mathrm{ICosDSinD}-\left(v_{\mathrm{e}}-\mathrm{i} \omega\right) \omega_{\mathrm{ce}} \operatorname{Sin} \mathrm{I}\right]}{\left[\left(v_{\mathrm{e}}-\mathrm{i} \omega\right)^{2}+\omega_{\mathrm{ce}}^{2}\right]} \mathrm{E}_{\mathrm{y}} \\
& +\frac{\sigma_{0}^{\prime}\left[-\omega_{\mathrm{ce}}^{2} \operatorname{CosISinISinD}-\left(v_{\mathrm{e}}-i \omega\right) \omega_{\mathrm{ce}} \operatorname{CosISinD}\right]}{\left[\left(v_{\mathrm{e}}-\mathrm{i} \omega\right)^{2}+\omega_{\mathrm{ce}}^{2}\right]} \mathrm{E}_{\mathrm{z}}
\end{aligned}
$$


By the same way, y direction the current density is resolved as follows:

$$
J_{y}=\frac{\operatorname{det} A_{2}}{\operatorname{det} A}=\frac{\sigma_{0}^{\prime}(a+b c)}{1+a^{2}+b^{2}+c^{2}} E_{x}+\frac{\sigma_{0}^{\prime}\left(1+b^{2}\right)}{1+a^{2}+b^{2}+c^{2}} E_{y}+\frac{\sigma_{0}^{\prime}(c-a b)}{1+a^{2}+b^{2}+c^{2}} E_{z}
$$

and the $\mathrm{J}_{\mathrm{y}}$ term is obtained as follows:

$$
\begin{aligned}
& \mathrm{J}_{\mathrm{y}}=\frac{\sigma_{0}^{\prime}\left[\left(v_{\mathrm{e}}-\mathrm{i} \omega\right) \omega_{\mathrm{ce}} \operatorname{Sin} \mathrm{I}+\omega_{\mathrm{ce}}^{2} \operatorname{Cos}^{2} \mathrm{ICosDSinD}\right]}{\left[\left(v_{\mathrm{e}}-\mathrm{i} \omega\right)^{2}+\omega_{\mathrm{ce}}^{2}\right]} \mathrm{E}_{\mathrm{x}}+\frac{\sigma_{0}^{\prime}\left[\left(v_{\mathrm{e}}-\mathrm{i} \omega\right)^{2}+\omega_{\mathrm{ce}}^{2} \operatorname{Cos}^{2} \mathrm{ICos}^{2} \mathrm{D}\right]}{\left[\left(v_{\mathrm{e}}-\mathrm{i} \omega\right)^{2}+\omega_{\mathrm{ce}}^{2}\right]} \mathrm{E}_{\mathrm{y}} \\
& +\frac{\sigma_{0}^{\prime}\left[\left(v_{\mathrm{e}}-i \omega\right) \omega_{\mathrm{ce}} \operatorname{Cos} I \operatorname{Sin} D-\omega_{\mathrm{ce}}^{2} \operatorname{Cos} \operatorname{Sin} I \operatorname{Cos} \mathrm{D}\right]}{\left[\left(v_{\mathrm{e}}-\mathrm{i} \omega\right)^{2}+\omega_{\mathrm{ce}}^{2}\right]} \mathrm{E}_{\mathrm{z}}
\end{aligned}
$$

Likely, $\mathrm{z}$ direction the current density is resolved as follows:

$$
J_{z}=\frac{\operatorname{det}_{3}}{\operatorname{det} \mathrm{A}}=\frac{\sigma_{0}^{\prime}(b-a c)}{1+a^{2}+b^{2}+c^{2}} E_{x}+\frac{\sigma_{0}^{\prime}(-c-a b)}{1+a^{2}+b^{2}+c^{2}} E_{y}+\frac{\sigma_{0}^{\prime}\left(1+a^{2}\right)}{1+a^{2}+b^{2}+c^{2}} E_{z}
$$

and the $\mathrm{J}_{\mathrm{z}}$ term is obtained as follows:

$$
\begin{aligned}
J_{z}= & \frac{\sigma_{0}^{\prime}\left[\left(v_{\mathrm{e}}-i \omega\right) \omega_{\mathrm{ce}} \operatorname{Cos} I \operatorname{Cos} D-\omega_{\mathrm{ce}}^{2} \operatorname{Cos} I \operatorname{Sin} I \operatorname{Sin} D\right]}{\left[\left(v_{\mathrm{e}}-\mathrm{i} \omega\right)^{2}+\omega_{\mathrm{ce}}^{2}\right]} \mathrm{E}_{\mathrm{x}} \\
& +\frac{\sigma_{0}^{\prime}\left[-\left(v_{\mathrm{e}}-i \omega\right) \omega_{\mathrm{ce}} \operatorname{Cos} I \operatorname{Sin} D-\omega_{\mathrm{ce}}^{2} \operatorname{Cos} \operatorname{Sin} I \operatorname{Cos} D\right]}{\left[\left(v_{\mathrm{e}}-i \omega\right)^{2}+\omega_{\mathrm{ce}}^{2}\right]} \mathrm{E}_{\mathrm{y}}+\frac{\sigma_{0}^{\prime}\left[\left(v_{\mathrm{e}}-i \omega\right)^{2}+\omega_{\mathrm{ce}}^{2} \operatorname{Sin}^{2} \mathrm{I}\right]}{\left[\left(v_{\mathrm{e}}-i \omega\right)^{2}+\omega_{\mathrm{ce}}^{2}\right]} \mathrm{E}_{\mathrm{z}}
\end{aligned}
$$

After being current densities obtained in this manner, $\mathrm{J}_{\mathrm{x}}, \mathrm{J}_{\mathrm{y}}$ and $\mathrm{J}_{\mathrm{z}}$ terms can be edited again by considering the $\sigma_{1}^{\prime}$ an $\sigma_{2}^{\prime}$ conductivities. Also, $\mathrm{J}_{\mathrm{x}}, \mathrm{J}_{\mathrm{y}}$ and $\mathrm{J}_{\mathrm{z}}$ terms can be written in the form of tensor like the following:

$$
\left[\begin{array}{l}
\mathbf{J}_{\mathrm{x}} \\
\mathbf{J}_{\mathrm{y}} \\
\mathrm{J}_{\mathrm{z}}
\end{array}\right]=\left[\begin{array}{ccc}
\sigma_{11} & \sigma_{12} & \sigma_{13} \\
\sigma_{21} & \sigma_{22} & \sigma_{23} \\
\sigma_{31} & \sigma_{32} & \sigma_{33}
\end{array}\right] \cdot\left[\begin{array}{c}
E_{x} \\
E_{y} \\
E_{z}
\end{array}\right]
$$

The conductivity tensor can defined as in Equation (47):

$$
\sigma^{\prime \prime}=\left[\begin{array}{lll}
\sigma_{11} & \sigma_{12} & \sigma_{13} \\
\sigma_{21} & \sigma_{22} & \sigma_{23} \\
\sigma_{31} & \sigma_{32} & \sigma_{33}
\end{array}\right]
$$

and tensor components can be achieved in a simpler as follows $[9,10]$ : 


$$
\begin{aligned}
& \sigma_{11}=\sigma_{1}^{\prime}+\left(\sigma_{0}^{\prime}-\sigma_{1}^{\prime}\right) \operatorname{Cos}^{2} \mathrm{I} \operatorname{Sin}^{2} \mathrm{D} \\
& \sigma_{12}=-\sigma_{2}^{\prime} \operatorname{Sin} I+\left(\sigma_{0}^{\prime}-\sigma_{1}^{\prime}\right) \operatorname{Cos}^{2} \mathrm{ICos} D \operatorname{Sin} \mathrm{D} \\
& \sigma_{13}=-\sigma_{2}^{\prime} \operatorname{CosICos} \mathrm{D}-\left(\sigma_{0}^{\prime}-\sigma_{1}^{\prime}\right) \operatorname{Cos} I \operatorname{SinISin} \mathrm{D} \\
& \sigma_{21}=\sigma_{2}^{\prime} \operatorname{SinI}+\left(\sigma_{0}^{\prime}-\sigma_{1}^{\prime}\right) \operatorname{Cos}^{2} \mathrm{ICos} \mathrm{DSinD} \\
& \sigma_{22}=\sigma_{1}^{\prime}+\left(\sigma_{0}^{\prime}-\sigma_{1}^{\prime}\right) \operatorname{Cos}^{2} \mathrm{ICos}^{2} \mathrm{D} \\
& \sigma_{23}=\sigma_{2}^{\prime} \operatorname{Cos} I \operatorname{Sin} D-\left(\sigma_{0}^{\prime}-\sigma_{1}^{\prime}\right) \operatorname{Cos} I \operatorname{SinI} \operatorname{Cos} \mathrm{D} \\
& \sigma_{31}=\sigma_{2}^{\prime} \operatorname{CosICosD}-\left(\sigma_{0}^{\prime}-\sigma_{1}^{\prime}\right) \operatorname{Cos} I \operatorname{Sin} I \operatorname{Sin} D \\
& \sigma_{32}=-\sigma_{2}^{\prime} \operatorname{CosISinD}-\left(\sigma_{0}^{\prime}-\sigma_{1}^{\prime}\right) \operatorname{Cos} I \operatorname{SinI} \operatorname{Cos} \mathrm{D} \\
& \sigma_{33}=\sigma_{1}^{\prime}+\left(\sigma_{0}^{\prime}-\sigma_{1}^{\prime}\right) \operatorname{Sin}^{2} \mathrm{I}
\end{aligned}
$$

\section{Dielectric constant for ionospheric plasma}

Dielectric constant for ionospheric plasma could be founded by using Maxwell equations.

1)

$$
\nabla \cdot \mathbf{E}=4 \pi \rho=\frac{\rho}{\varepsilon_{0}}
$$

2)

$$
\nabla \cdot \mathbf{B}=0
$$

3)

$$
\nabla \times \mathbf{E}=-\frac{\partial \mathbf{B}}{\partial \mathrm{t}}
$$

4)

$$
\nabla \times \mathbf{B}=\frac{1}{\mathrm{c}^{2}} \frac{\partial \mathbf{E}}{\partial \mathrm{t}}+\mu_{0} \mathbf{J}
$$

Where $\mathrm{c}=\frac{1}{\sqrt{\mu_{0} \varepsilon_{0}}}=3 \times 10^{8}$ is the light speed. According to fourth Maxwell equation,

$$
\nabla \times \mathbf{B}=\mu_{0} \varepsilon_{0} \frac{\partial \mathbf{E}}{\partial \mathrm{t}}+\mu_{0} \mathbf{J}
$$

and if the electric field is accepted the form change $\mathrm{e}^{-\mathrm{i} \omega \mathrm{t}}$ then,

$$
\nabla \times \mathbf{B}=\mu_{0} \varepsilon_{0} \frac{\partial}{\partial \mathrm{t}}\left(\mathbf{E}_{0} \mathrm{e}^{-\mathrm{i} \omega \mathrm{t}}\right)+\mu_{0} \mathbf{J}
$$

From here 


$$
\nabla \times \mathbf{B}=-i \omega \mu_{0} \varepsilon_{0}\left[\mathbf{E}-\frac{\sigma \mathbf{E}}{i \omega \varepsilon_{0}}\right]
$$

it is obtained as follow

$$
\nabla \times \mathbf{B}=-\mathrm{i} \omega \mu_{0} \varepsilon_{0}\left[1-\frac{\sigma}{\mathrm{i} \omega \varepsilon_{0}}\right] \mathbf{E}
$$

or

$$
\nabla \times \mathbf{B}=-\mathrm{i} \omega \mu_{0} \mathbf{E}\left[\varepsilon_{0}\left(1-\frac{\sigma}{\mathrm{i} \omega \varepsilon_{0}}\right)\right]
$$

According to the latest's equation, the dielectric constant of any medium

$$
\varepsilon=\varepsilon_{0}\left(1-\frac{\sigma}{i \omega \varepsilon_{0}}\right)
$$

In which, because of $(\sigma)$ the tensorial form $\underset{\sim}{1}$ is the unit tensor.

$$
\underset{\sim}{1}=\left[\begin{array}{lll}
1 & 0 & 0 \\
0 & 1 & 0 \\
0 & 0 & 1
\end{array}\right]
$$

Generally, the expression of ionospheric conductivity $\sigma^{\prime \prime}$ given in Equation (47) by using Equation (57), the dielectric structure of ionospheric plasma is shown by

$$
\varepsilon=\varepsilon_{0}\left[\left[\begin{array}{lll}
1 & 0 & 0 \\
0 & 1 & 0 \\
0 & 0 & 1
\end{array}\right]-\frac{1}{i \omega \varepsilon_{0}}\left[\begin{array}{ccc}
\sigma_{11} & \sigma_{12} & \sigma_{13} \\
\sigma_{21} & \sigma_{22} & \sigma_{23} \\
\sigma_{31} & \sigma_{32} & \sigma_{33}
\end{array}\right]\right]
$$

\section{The refractive index of the cold plasma}

The refractive index (n) determines the behavior of electromagnetic wave in a medium and refractive index of the medium is founded by using Maxwell equations. If the curl $(\nabla \times)$ of the Equation (50) is taken,

$$
\nabla \times \nabla \times \mathbf{E}=-\frac{\partial}{\partial \mathrm{t}}(\nabla \times \mathbf{B})
$$

If $(\nabla \times \mathbf{B})$ term in this expression is re-written in (60),

$$
\nabla \times \nabla \times \mathbf{E}=\mu_{0} \varepsilon_{0} \omega^{2}\left[\underset{\sim}{1}+\frac{i \sigma}{\varepsilon_{0} \omega}\right] \cdot \mathbf{E}
$$


Since the electric field $\mathbf{E}$ varies as $\mathrm{e}^{\mathrm{i}(\mathbf{k} \cdot \mathbf{r}-\omega \mathrm{t})}$, it can be assumed that $\nabla=\mathrm{ik}$. In this case, the left side of the Equation (61) can be defined as follows:

$$
\nabla \times \nabla \times \mathbf{E}=\mathrm{k}^{2} \mathbf{E}-\mathbf{k}(\mathbf{k} \cdot \mathbf{E})
$$

If the Equations (61) and (62) are rearranged, then Equation (63) can be defined as follows:

$$
\mathrm{k}^{2} \mathbf{E}-\mathbf{k}(\mathbf{k} \cdot \mathbf{E})=\frac{\omega^{2}}{\mathrm{c}^{2}}\left[\underset{\sim}{1+}+\frac{\mathrm{i} \sigma}{\varepsilon_{0} \omega}\right] \cdot \mathbf{E}
$$

If the wave vector $\mathrm{k}$ in this equation is written in terms of refractive index $\mathbf{n}$, the Equation (63) can be defined in terms of refractive index as follows:

$$
\begin{gathered}
\mathbf{k}=\frac{\omega}{\mathrm{c}} \mathbf{n} \\
\mathrm{n}^{2} \mathbf{E}-\mathbf{n}(\mathbf{n} \cdot \mathbf{E})=\left[\underset{\sim}{1+\frac{\mathrm{i} \sigma}{\varepsilon_{0} \omega}}\right] \cdot \mathbf{E}
\end{gathered}
$$

If the necessary procedures are used, the Equation (66) is obtained as follows:

$$
\left[\begin{array}{ccc}
\mathrm{n}^{2} & 0 & 0 \\
0 & \mathrm{n}^{2} & 0 \\
0 & 0 & 0
\end{array}\right] \cdot\left[\begin{array}{l}
\mathrm{E}_{\mathrm{x}} \\
\mathrm{E}_{\mathrm{y}} \\
\mathrm{E}_{\mathrm{z}}
\end{array}\right]=\left[\begin{array}{ccc}
1 & 0 & 0 \\
0 & 1 & 0 \\
0 & 0 & 1
\end{array}\right]+\frac{\mathrm{i}}{\varepsilon_{0} \omega}[\sigma] \cdot\left[\begin{array}{c}
\mathrm{E}_{\mathrm{x}} \\
\mathrm{E}_{\mathrm{y}} \\
\mathrm{E}_{\mathrm{z}}
\end{array}\right]
$$

By writing the conductivity tensor $\sigma^{\prime \prime}$ in the Equation (47) instead of the conductivity in the Equation (66), a relation for the cold plasma is obtained as follows:

$$
\left[\begin{array}{ccc}
n^{2}-1-\frac{i \sigma_{11}}{\varepsilon_{0} \omega} & -\frac{i \sigma_{12}}{\varepsilon_{0} \omega} & -\frac{i \sigma_{13}}{\varepsilon_{0} \omega} \\
-\frac{i \sigma_{21}}{\varepsilon_{0} \omega} & n^{2}-1-\frac{i \sigma_{22}}{\varepsilon_{0} \omega} & -\frac{i \sigma_{23}}{\varepsilon_{0} \omega} \\
-\frac{i \sigma_{31}}{\varepsilon_{0} \omega} & -\frac{i \sigma_{32}}{\varepsilon_{0} \omega} & -1-\frac{i \sigma_{33}}{\varepsilon_{0} \omega}
\end{array}\right] \cdot\left[\begin{array}{l}
E_{\mathrm{x}} \\
E_{\mathrm{y}} \\
E_{\mathrm{z}}
\end{array}\right]=0
$$

Here, since the electric fields do not equal to zero, the determinant of the matrix of the coefficients equals to zero. So:

$$
\left[\begin{array}{ccc}
n^{2}-1-\frac{i \sigma_{11}}{\varepsilon_{0} \omega} & -\frac{i \sigma_{12}}{\varepsilon_{0} \omega} & -\frac{i \sigma_{13}}{\varepsilon_{0} \omega} \\
-\frac{i \sigma_{21}}{\varepsilon_{0} \omega} & n^{2}-1-\frac{i \sigma_{22}}{\varepsilon_{0} \omega} & -\frac{i \sigma_{23}}{\varepsilon_{0} \omega} \\
-\frac{i \sigma_{31}}{\varepsilon_{0} \omega} & -\frac{i \sigma_{32}}{\varepsilon_{0} \omega} & -1-\frac{i \sigma_{33}}{\varepsilon_{0} \omega}
\end{array}\right]=0
$$


The matrix given by Equation (68) includes the information about the propagation of the wave. Since the matrix has a complex structure, it is impossible to solve the matrix in general. However, the numerical analysis can be done for the matrix. Therefore, solutions should be made to certain conditions, in terms of convenience.

\section{$4.1 \mathrm{k} / / \mathrm{B}$ condition: plasma oscillation and polarized waves}

When the progress vector of the wave $(\mathbf{k})$ is parallel or anti-parallel to the earth's magnetic field or the any components of the earth's magnetic field, two cases can be observed for the ionospheric plasma depending on the refractive index of the medium. For example, if the wave propagates in the $\mathrm{z}$ direction as in the vertical ionosondas, the vertical component of the earth's magnetic field effects to the propagation of the wave. In this case, two waves occur from the solution of the determinant given by Equation (68). The first one is the vibration of the plasma defined as follows:

$$
\omega_{\mathrm{pe}}^{2}=\omega^{2}\left(1-\mathrm{n}^{2}\right)
$$

The second one is the polarized wave given by Equation (70) [9].

$$
\mathrm{n}_{\mathrm{p}}^{2}=1-\frac{\mathrm{X}(1 \mp \mathrm{Y})}{(1 \mp \mathrm{Y})^{2}+\mathrm{Z}^{2}}+\mathrm{iZ} \frac{\mathrm{X}}{(1 \mp \mathrm{Y})^{2}+\mathrm{Z}^{2}}
$$

The signs \pm in the Equation (70) represents the right-polarized (-) and left-polarized (+) waves, respectively. In this equation, $X=\frac{\omega_{\mathrm{pe}}^{2}}{\omega^{2}}, Y=\frac{\omega_{\mathrm{ce}}}{\omega}$ and $Z=\frac{v_{\mathrm{e}}}{\omega}$. The magnetic field in the expression $\mathrm{Y}$ is the cyclotron frequency caused by the $\mathrm{z}$ component of the earth's magnetic field $\left(Y=\frac{\omega_{\mathrm{ce}}}{\omega} \operatorname{SinI}\right)$. This equation is the complex expression. Since the refractive index of the medium determines the resonance $\left(n^{2} \cong \infty\right)$ and the cut-off $\left(n^{2}=0\right)$ conditions of the wave, it is the most important parameter in the wave studies. If it is taken that $Z=0$, the equation becomes simple. The resonance and the reflection conditions of the polarized wave become different from the case of $Z \neq 0$.

\section{$4.2 \mathrm{k} \perp \mathrm{B}$ condition: ordinary and extra-ordinary waves}

When the propagation vector of the wave (k) is perpendicular to the earth's magnetic field, two waves occur in the ionospheric plasma [9]. The first wave is the ordinary wave given as follows:

$$
\mathrm{n}_{\mathrm{o}}^{2}=1-\frac{\mathrm{X}}{1+\mathrm{Z}^{2}}+\mathrm{iZ} \frac{\mathrm{X}}{1+\mathrm{Z}^{2}}
$$

This wave does not depend on Earth's magnetic field. However it depends on the collisions. The collisions can change the resonance and the reflection frequencies of the wave. The second wave is the extra ordinary wave depending on the magnetic field. The refractive index of the extra ordinary wave can be defined as follows: 


$$
\mathrm{n}_{\mathrm{ex}}^{2}=1-\frac{\mathrm{aX}(1-X)+\mathrm{Z}^{2} \mathrm{X}(2-\mathrm{X})}{\mathrm{a}^{2}+\mathrm{b}^{2}}+\mathrm{iZ} \frac{\mathrm{X}(1-\mathrm{X})(2-\mathrm{X})-\mathrm{aX}}{\mathrm{a}^{2}+\mathrm{b}^{2}}
$$

Where $a=1-X-Y^{2}-Z^{2}$ and $b=Z(2-X)$. This relation is valid for the $y$ direction. So, the magnetic field in the $\mathrm{Y}$ is the cyclotron frequency caused by the $\mathrm{y}$ component of the earth's magnetic field. By the same way, the wave defined by this relation is also observed in the $x$ direction. For the extra ordinary wave in the $x$ direction, the cyclotron frequency, that is contained in $\mathrm{Y}$, is the cyclotron frequency caused by the $\mathrm{x}$ component of the earth's magnetic field.

4.3 The Binom expansion $\left(\left(1+Z^{2}\right)^{-1} \approx 1-Z^{2}\right)$ and the refractive indices

The solutions of the refractive indices mentioned above are complex and difficult. The solutions can be obtained by using the binom expansion. Accordingly, the refractive indices can be obtained by using the Binom expansion and the real part of refractive indices as follows:

1. For the right-polarized wave given by Equation (70):

$$
\begin{gathered}
\mu_{\mathrm{p}}^{2} \approx\left(1-X^{\prime}\right)+Z^{\prime 2} \frac{X^{\prime}\left(4-3 X^{\prime}\right)}{4\left(1-X^{\prime}\right)} \quad \text { for } X^{\prime}\langle 1 \\
\mu_{\mathrm{p}}^{2} \approx Z^{\prime 2} \frac{X^{\prime} 2}{4\left(X^{\prime}-1\right)} \quad \text { for } \quad X^{\prime}>1
\end{gathered}
$$

Where, $\quad X^{\prime}=\frac{X}{1-Y \operatorname{SinI}}$ and $Z^{\prime}=\frac{Z}{1-Y \operatorname{SinI}}$.

2. For the extra ordinary wave given by Equation (71):

$$
\begin{gathered}
\mu_{\mathrm{o}}^{2} \approx(1-X)+Z^{2} \frac{X(4-3 X)}{4(1-X)} \text { for } X\langle 1 \\
\mu_{\mathrm{o}}^{2} \approx Z^{2} \frac{X^{2}}{4(X-1)} \quad \text { for } X>1
\end{gathered}
$$

3. For the extra ordinary wave given by Equation (72):

$$
\begin{aligned}
\mu_{\mathrm{ex}}^{2} & \approx \frac{(1-X)^{2}-\mathrm{Y}^{2} \operatorname{Cos}^{2} I \operatorname{Cos}^{2} \mathrm{D}}{1-\mathrm{X}-\mathrm{Y}^{2} \operatorname{Cos}^{2} \mathrm{I} \operatorname{Cos}^{2} \mathrm{D}} \\
& +\mathrm{Z}^{2} \frac{\mathrm{X}^{2}\left[(1-X)^{2}+\mathrm{Y}^{2} \operatorname{Cos}^{2} \mathrm{I} \operatorname{Cos}^{2} \mathrm{D}\right]^{2}}{4\left[1-X-\mathrm{Y}^{2} \operatorname{Cos}^{2} \mathrm{I} \operatorname{Cos}^{2} \mathrm{D}\right]^{3}\left[(1-X)^{2}-\mathrm{Y}^{2} \operatorname{Cos}^{2} \mathrm{I} \operatorname{Cos}^{2} \mathrm{D}\right]}
\end{aligned}
$$




\section{Relaxation mechanism of cold ionospheric plasma}

\subsection{Charge conservation}

Maxwell added the displacement current to Ampere law in order to guarantee charge conversation. Indeed, talking the divergence of both sides of Ampere's law and using Gauss's law $\nabla \cdot \mathbf{D}=\rho$, we get:

$$
\nabla \cdot \nabla \times \mathbf{H}=\nabla \cdot \mathbf{J}+\nabla \cdot \frac{\partial \mathbf{D}}{\partial \mathrm{t}}=\nabla \cdot \mathbf{J}+\frac{\partial}{\partial \mathrm{t}} \nabla \cdot \mathbf{D}=\nabla \cdot \mathbf{J}+\frac{\partial \rho}{\partial \mathrm{t}}
$$

Using the vector identity $\nabla \cdot \nabla \times \mathbf{H}=0$, we obtain the differential from of the charge conversation law:

$$
\nabla \cdot \mathbf{J}+\frac{\partial \rho}{\partial \mathrm{t}}=0 \text { (charge conservation) }
$$

Integrating both sides over a closed volume V surrounded by the surface S, and using the divergence theorem, we obtain the integrated

$$
\oint_{S} \mathbf{J} \cdot \mathrm{d} \mathbf{S}=-\frac{\mathrm{d}}{\mathrm{dt}} \int_{\mathrm{V}} \rho \mathrm{dV}
$$

The left-hand represents the total amount of charge flowing outwards through the surface $S$ per unit time. The right-hand side represents the amount by which the charge is decreasing inside the volume $\mathrm{V}$ per unit time. In other words, charge does not disappear into (or get created out of) nothingness-it decreases in a region of space only because it flows into other regions.

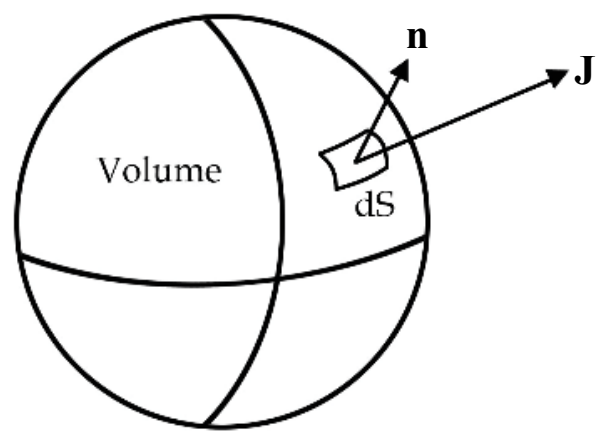

Fig. 7. Flux outwards through surface

Another consequence is that in good conductors, there cannot be any accumulated volume charge. Any such charge will quickly move to the conductor's surface and distribute itself such that to make the surface into an equipotential surface. Assuming that inside the conductor we have $\mathbf{D}=\varepsilon \cdot \mathbf{E}$ and $\mathbf{J}=\sigma \cdot \mathbf{E}$, we obtain

$$
\nabla \cdot \mathbf{J}=\sigma \nabla \cdot \mathbf{E}=\frac{\sigma}{\varepsilon} \nabla \cdot \mathbf{D}=\frac{\sigma}{\varepsilon} \rho
$$


Therefore,

$$
\frac{\mathrm{d} \rho}{\mathrm{dt}}+\frac{\sigma}{\varepsilon} \rho=0
$$

with solution:

$$
\rho(\mathbf{r}, \mathrm{t})=\rho_{0}(\mathbf{r}) \mathrm{e}^{-\sigma t / \tau}
$$

Where, $\rho_{0}(\mathbf{r})$ is the initial volume charge distribution. The solution shows that the volume charge disappears from inside and therefore it must accumulate on the surface of the conductors. For example, in copper,

$$
\tau_{\text {rel }}=\frac{\varepsilon}{\sigma}=\frac{8.85 \times 10^{-12}}{5.7 \times 10^{7}}=1.6 \times 10^{-19} \mathrm{sec}
$$

By contrast, $\tau_{\text {rel }}$ is of the order of days in a good dielectric. For good conductors, the above argument is not quite correct because it is based on the steady-state version of Ohm's law, which must be modified to take into account the transient dynamics of the conduction charges.

It turn out that the relaxation time $\tau_{\text {rel }}$ is of the collision time, which is typically $10^{-14} \mathrm{sec}$.

\subsection{Charge relaxation in conductors}

We discuss the issue of charge relaxation in good conductors. Writing three-dimensionally and using, Ohm's law reads in the time domain:

$$
J(r, t)=\omega_{p e}^{2} \int_{-\infty}^{t} e^{-\alpha\left(t-t^{\prime}\right)} \varepsilon_{0} E\left(r, t^{\prime}\right) d t^{\prime}
$$

Taking the divergence of both sides and using charge conversation, $\nabla \cdot \mathbf{J}+\dot{\rho}=0$, and Gauss's law, $\varepsilon_{0} \boldsymbol{\nabla} \cdot \mathbf{E}=\rho$, we obtain the following integro-differential equation fort he charge density $\rho(\mathbf{r}, \mathbf{t})$ :

$$
-\dot{\rho}(\mathbf{r}, \mathrm{t})=\nabla \cdot \mathbf{J}(\mathbf{r}, \mathrm{t})=\omega_{\mathrm{pe}}^{2} \int_{-\infty}^{\mathrm{t}} \mathrm{e}^{-\alpha\left(\mathrm{t}-\mathrm{t}^{\prime}\right)} \varepsilon_{0} \nabla \cdot \mathbf{E}\left(\mathbf{r}, \mathrm{t}^{\prime}\right) d \mathrm{t}^{\prime}=\omega_{\mathrm{pe}}^{2} \int_{-\infty}^{\mathrm{t}} \mathrm{e}^{-\alpha\left(\mathrm{t}-\mathrm{t}^{\prime}\right)} \rho\left(\mathbf{r}, \mathrm{t}^{\prime}\right) d \mathrm{t}^{\prime}
$$

Differentiating both sides with respect to, we find that $\rho$ satisfies the second-order differential equation:

$$
\ddot{\rho}(\mathbf{r}, \mathrm{t})+\alpha \dot{\rho}(\mathbf{r}, \mathrm{t})+\omega_{\text {pe }}^{2} \rho(\mathbf{r}, \mathrm{t})=0
$$

whose solution is easily verified to be a linear combination of:

$$
e^{-\alpha t / 2} \operatorname{Cos}\left(\omega_{\text {rel }} t\right), e^{-\alpha t / 2} \operatorname{Sin}\left(\omega_{\text {rel }} t\right)
$$

Where $\omega_{\mathrm{rel}}=\sqrt{\omega_{\mathrm{pe}}^{2}-\frac{\alpha^{2}}{4}}$. Thus the charge density is an exponentially decaying sinusoid with a relaxation time constant that is twice the collision time $\tau=1 / \alpha$ : 


$$
\tau_{\text {rel }}=\frac{2}{\alpha}=2 \tau \text { (relaxation time constant) }
$$

Typically, $\left.\left.\omega_{\mathrm{pe}}\right\rangle\right\rangle \alpha$, so that $\omega_{\text {rel }}$ is practically equal to $\omega_{\mathrm{pe}}$. For example, using the numerical data of example, we find for copper $\tau_{\text {rel }}=2 \tau=5 \times 10^{-14} \mathrm{sec}$. We calculate also: $\mathrm{f}_{\mathrm{el}}=\omega_{\mathrm{rel}} / 2 \pi=2.6 \times 10^{-15} \mathrm{~Hz}$. In the limit $\alpha \rightarrow \infty$ or $\tau \rightarrow 0$, reduces to the naive relaxation.

In addition to charge relaxation, the total time depends on the time it takes fort he electric and magnetic fields to be extinguished from the inside of the conductor, as well as the time it takes fort he accumulated surface charge densities to setle, the motion of the surface charges being damped because of ohmic losses. Both of these times depend on the geometry and size of the conductor. The components of conductivity have given by Equation (47) and the permittivity of plasma has given by Equation (59).

As the conductivity of plasma has a tensor form, the permittivity of plasma is also a tensor. Moreover, the permittivity of plasma is frequency dependent and each component of the permittivity tensor has real and imaginer part. Consequently, the permittivity of plasma depends on the conductivity of plasma. The permittivity of plasma could be expressed as follow.

$$
\varepsilon=\left[\begin{array}{lll}
\varepsilon_{11} & \varepsilon_{12} & \varepsilon_{13} \\
\varepsilon_{21} & \varepsilon_{22} & \varepsilon_{23} \\
\varepsilon_{31} & \varepsilon_{32} & \varepsilon_{33}
\end{array}\right]
$$

The real and imaginary part of the permittivity is an indication of the ohmic power loss. Anisotropy is an inherent property of the atomic/molecular structure of the dielectric. It may also be caused by the application of external fields. For example, conductors and plasmas in the presence of a constant magnetic field -such as the ionosphere in the presence of the Earth's magnetic field- become anisotropic. The relaxation time in the ionospheric plasma has tensorial form as follow.

$$
\tau=\left[\begin{array}{lll}
\tau_{11} & \tau_{12} & \tau_{13} \\
\tau_{21} & \tau_{22} & \tau_{23} \\
\tau_{31} & \tau_{32} & \tau_{33}
\end{array}\right]
$$

The relaxation time in the ionospheric plasma is different at every direction and have real and imaginary parts [11].

\section{Ionospheric absorption and attenuation of radio wave}

When the radio waves propagate in the ionospheric plasma, they exhibit different behaviors related to their wave frequency, oscillation frequency of the electrons in the plasma medium and the refractive index of the medium. Depending on these behaviors, the wave is refracted, reflected or attenuated by absorption from medium. Radio-wave damping is due to movements in the ionosphere of electrons and ions are caused by collisions with other particles [12]. Due to the increase of collisions, absorption increases and field strength of the radio-wave decreases. As a result of this, amplitude of the radio-wave propagated in the ionosphere will decrease because of the absorption. Thus, the real part of the refractive 
index effects to the phase velocity and the imaginary part of the refractive index is associated with spatial attenuation of the wave.

In accordance with the above information, in order to obtain the relation of the attenuation, amplitude of the wave, such as the electric field strength, is need to be expressed depending on the refractive index of the medium. Accordingly, the electric field strength of the wave can be defined as follows:

$$
E=E_{0} e^{\mp i(k \cdot r-\omega t)}
$$

Here, the wave vector $\mathbf{k}$ is written in terms of refractive index $\mathbf{n}$, Equation (92) becomes as follows:

$$
\mathbf{E}=\mathbf{E}_{0} \mathrm{e}^{\mp \mathrm{i}\left(\frac{\omega}{\mathrm{c}} \mathbf{n} \cdot \mathbf{r}-\omega \mathrm{t}\right)}
$$

The refractive index in the ionospheric plasma is also defined as in equation (93).

$$
\mathrm{n}=\mu+\mathrm{i} \chi
$$

where, $\mu$ and $\chi$ represent the real and the imaginary part of the refractive index, respectively. The collision of the electron with the other particles effects to the real and the imaginary part of the refractive index. In the High Frequency $(\mathrm{HF})$ waves, $\mathrm{Z}$ is very smaller than $1\left(Z\left\langle\langle 1)\right.\right.$. Therefore, $Z$ can be defined as $\left(1+Z^{2}\right)^{-1} \approx 1-Z^{2}$. The real and the imaginary part of the refractive index and the phase velocity of the polarized ordinary and extraordinary wave can be determined by using this evolution.

Accordingly, if the refractive index given by Equation (94) is written in Equation (93), the electric field strength can be obtained as follows:

$$
\mathbf{E}=\mathbf{E}_{0} \mathrm{e}^{\mp \mathrm{i}\left(\frac{\omega}{\mathrm{c}} \mu \mathrm{r}-\omega \mathrm{t}\right)} \mathrm{e}^{-\frac{\omega}{\mathrm{c}} \chi \mathrm{r}}
$$

The part of the damping in the electric field strength in Equation (95) is the exponential term related to second $\chi$ in the right side of the equation. Thus, the attenuation of the wave is represented by $\chi$. According to this, the refractive indexes of the polarized, ordinary and extra-ordinary waves given (70)-(72) equations for cold plasma could be showed as the real and imaginary parts as follow.

$$
\mathrm{n}^{2}=\mathrm{F}+\mathrm{iG}
$$

Accordingly, the electric field strength given in Equation (95) can be defined as follows:

$$
\mathbf{E}=\mathbf{E}_{0} \mathrm{e}^{\mp \mathrm{i}\left(\frac{\omega}{\mathrm{c}} \mu \mathrm{r}-\omega \mathrm{t}\right)} \mathrm{e}^{-\frac{\omega}{\mathrm{c}}\left[\frac{ \pm\left(\mathrm{F}^{2}+\mathrm{G}^{2}\right)^{1 / 2}-\mathrm{F}}{2}\right]^{1 / 2} \mathrm{r}}
$$

In the Equation (97), signs (+) and (-) in front of the first exponential expression represents the wave propagated to upward $(+\hat{z})$ and downward $(-\hat{z})$ after the reflection, respectively. 
Here, by considering the electromagnetic wave that propagated to upward $+\hat{z}$, the term $\mathrm{e}^{-\frac{\omega}{\mathrm{c}} x \mathrm{r}}$ can be defined as $\mathrm{e}^{-\frac{\omega}{\mathrm{c}} \chi z}$. Therefore, the damping term of the wave amplitude is defined with the height as follows:

$$
\mathbf{E}=\mathbf{E}_{0} \mathrm{e}^{\mp i\left(\frac{\omega}{\mathrm{c}} \mu \mathrm{z}-\omega \mathrm{t}\right)} e^{-\frac{\omega}{\mathrm{c}}\left[\frac{ \pm\left(\mathrm{F}^{2}+\mathrm{G}^{2}\right)^{1 / 2}-\mathrm{F}}{2}\right]^{1 / 2} \mathrm{z}}
$$

The second term in equation (98) represents how much the attenuation of wave in ionospheric plasma.

\section{Conclusion}

Ionospheric plasma has double-refractive index and generally weak conductivity in every direction, season and local time. Due to these, the ionospheric parameters such as conductivity, dielectric constant and the refractive index are different in every direction and have the complex structure (both the real and imaginary part). This shows that the diagonal elements of conductivity, refractive index and dielectric tensor which the conductivities dominate have generally higher conductivity than other elements; besides any electromagnetic wave propagating in ionospheric plasma could be sustained attenuation in every direction. So this attenuation results from the imaginary part of the refractive index.

\section{Symbol list}

\begin{tabular}{|c|c|}
\hline $\mathrm{m}_{\mathrm{e}}$ & : Mass of electron \\
\hline $\mathrm{m}_{\mathrm{i}}$ & : Mass of ion \\
\hline $\mathbf{V}_{\mathrm{e}}$ & : Velocity of electron \\
\hline $\mathrm{t}$ & : Time \\
\hline -e & : Charge of electron \\
\hline E & : Electric field intensity \\
\hline B & : Magnetic induction \\
\hline$v_{\mathrm{e}}$ & : Electron collision frequency \\
\hline$v_{\mathrm{ei}}$ & : Electron-ion collision frequency \\
\hline$v_{\mathrm{en}}$ & : Electron-neutral particle collision frequency \\
\hline J & : Current density \\
\hline $\mathrm{N}_{\mathrm{e}}$ & : Electron density \\
\hline$\sigma$ & : Conductivity \\
\hline$\omega_{\mathrm{ce}}$ & : Electron cyclotron frequency \\
\hline$\omega$ & : Wave angular frequency \\
\hline$\omega_{\mathrm{pe}}$ & : Electron plasma frequency \\
\hline$\varepsilon_{0}$ & : Dielectric constant of free space \\
\hline I & : Dip angle \\
\hline $\mathrm{D}$ & : Declination angle \\
\hline$\nabla$ & : Dell operator \\
\hline
\end{tabular}




$\begin{array}{ll}\rho & : \text { Charge density } \\ \mu_{0} & : \text { Magnetic permeability of free space } \\ \mathrm{c} & : \text { Speed of light } \\ \varepsilon & : \text { Dielectric constant of medium } \\ 1 & : \text { Unit tensor } \\ \mathbf{k} & : \text { Electromagnetic wave vector } \\ \mathbf{r} & : \text { Displacement vector } \\ \mathbf{n} & : \text { Refractive index } \\ \mu & : \text { Real part of refractive index } \\ \mu_{\mathrm{p}} & : \text { Real part of refractive index of polarized wave } \\ \mu_{\mathrm{o}} & : \text { Real part of refractive index of ordinary wave } \\ \mu_{\mathrm{ex}} & : \text { Real part of refractive index of extra-ordinary wave } \\ \mathrm{D} & : \text { Displacement current } \\ \mathrm{H} & : \text { Magnetic field intensity } \\ \mathrm{S} & : \text { Surface } \\ \mathrm{V} & : \text { Volume } \\ \tau & : \text { Collision time } \\ \tau_{\mathrm{rel}} & : \text { Relaxation time } \\ \mathrm{a} & : \text { The measure of the rate of collisions per unit time } \\ \mathrm{f} & : \text { Linear frequency } \\ \chi & : \text { Imaginary part of refractive index }\end{array}$

\section{References}

[1] H. Rishbeth, A reviev of ionospheric F region theory, Proc. of the IEEE., Vol.55(1), pp.1635, 1967.

[2] H. Rishbeth and M. Mendillo, Patterns of F2-layer variability, J. Atmos. Solar-Terr. Phys., Vol.63(15), pp.1661-1680, 2001.

[3] M. Grabner and V. Kvicera, Refractive index measurements in the lowest troposphere in the Czech Republic, J. Atmos. Solar-Terr. Phys., Vol.68(12), pp.1334-1339, 2006.

[4] H. Rishbeth, Review paper:The ionospheric E layer and F layer dynamos -a tutorial review, J. Atmos. Solar-Terr. Phys., Vol.59(15), pp.1873-1880, 1997.

[5] R. Della and J. Devin, A graphical interpretation of the electrical conductivity tensor, J. Atmos. Solar-Terr. Phys., Vol.67(4), pp.337-343, 2005.

[6] H. G. Booker, Cold Plasma Waves. Martinus Nijhoff Publishers, Dordrecht-Netherlands, 1984.

[7] R. O. Dendy, Plasma Dynamics. Clarendon Press, Oxford, 1990.

[8] B. S. Tanenbaum, Plasma Physics. McGraw-Hill Book Company, New York 1967.

[9] M. Aydoğdu, A. Yeşil and E. Güzel, The group refractive indices of HF waves in the ionosphere and departure from the magnitude without collisions, J. Atmos. and Solar-Terr. Phys. Vol. 66(5), pp.343-348, 2004.

[10] M. Aydoğdu, E. Güzel, A. Yeşil, O. Özcan and M. Canyılmaz, Comparison of the calculated absorption and the measured field strength of HF waves reflected from the ionosphere, Il Nouovo Cimento, Vol.30C, No.3, pp.243-253, 2007. 
[11] A. Yeşil, and İ. Ünal, The effect of altitude and season dielectrical relaxation mechanism of ionospheric plasma, Il Nouovo Cimento, Vol.124B, No.7, pp.777-784, 2009.

[12] J. A. Ratcliffe, The Magneto-Ionic Theory and its Applications to the Ionosphere. Cambridge University Press, 1959. 


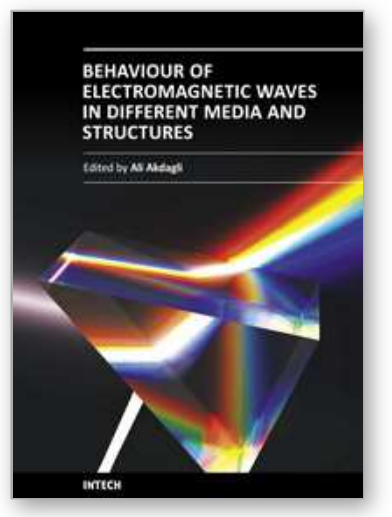

\section{Behaviour of Electromagnetic Waves in Different Media and Structures \\ Edited by Prof. Ali Akdagli}

ISBN 978-953-307-302-6

Hard cover, 440 pages

Publisher InTech

Published online 09, June, 2011

Published in print edition June, 2011

This comprehensive volume thoroughly covers wave propagation behaviors and computational techniques for electromagnetic waves in different complex media. The chapter authors describe powerful and sophisticated analytic and numerical methods to solve their specific electromagnetic problems for complex media and geometries as well. This book will be of interest to electromagnetics and microwave engineers, physicists and scientists.

\section{How to reference}

In order to correctly reference this scholarly work, feel free to copy and paste the following:

Ali Yesil and Ibrahim Unal (2011). Electromagnetic Wave Propagation in Ionospheric Plasma, Behaviour of Electromagnetic Waves in Different Media and Structures, Prof. Ali Akdagli (Ed.), ISBN: 978-953-307-302-6, InTech, Available from: http://www.intechopen.com/books/behavior-of-electromagnetic-waves-in-differentmedia-and-structures/electromagnetic-wave-propagation-in-ionospheric-plasma1

\section{INTECH}

open science | open minds

\author{
InTech Europe \\ University Campus STeP Ri \\ Slavka Krautzeka 83/A \\ 51000 Rijeka, Croatia \\ Phone: +385 (51) 770447 \\ Fax: +385 (51) 686166 \\ www.intechopen.com
}

\author{
InTech China \\ Unit 405, Office Block, Hotel Equatorial Shanghai \\ No.65, Yan An Road (West), Shanghai, 200040, China \\ 中国上海市延安西路65号上海国际贵都大饭店办公楼 405 单元 \\ Phone: +86-21-62489820 \\ Fax: $+86-21-62489821$
}


(C) 2011 The Author(s). Licensee IntechOpen. This chapter is distributed under the terms of the Creative Commons Attribution-NonCommercialShareAlike-3.0 License, which permits use, distribution and reproduction for non-commercial purposes, provided the original is properly cited and derivative works building on this content are distributed under the same license. 